Harjola ${ }^{5}$ and Dunbar. ${ }^{6}$ The outstanding symptom is upper abdominal pain after meals, usually intermittent but occasionally continuous. Loss of weight and diarrhoea-such prominent features of mesenteric ischaemia-are not particularly notable. The main diagnostic clue is a loud, localised systolic bruit in the epigastrium, ${ }^{7}$ and the diagnosis is established by angiography, when lateral aortography shows a smooth unfolding of the upper surface of a compressed coeliac axis with poststenotic dilatation. Herlinger ${ }^{8}$ has emphasised the importance of accurate tangential radiography if the full abnormality is to be seen in clear profile. The condition seems mainly to afflict women in their $20 \mathrm{~s}$ and $30 \mathrm{~s}$, and, though the nature of the constricting agent is uncertain, it is probably either the median arcuate ligament of the diaphragm ${ }^{6}$ or the neurofibrous tissue of the coeliac ganglion. ${ }^{9}$ Surgical release of this obstruction has abolished the symptoms or produced major benefit in most of the reported cases. Again, surgeons disagree about the operative procedure. Simply cutting the median arcuate ligament, with or without dividing or resecting the ganglion, is the common practice, but the presence of an organic stricture causing permanent stenosis of the coeliac axis ${ }^{10}$ has prompted some surgeons to advise arterial reconstruction. ${ }^{11}$

The latest paper, from Canada, ${ }^{12}$ reports a further 20 patients treated surgically over five years; most (about $80 \%$ ) obtained symptomatic relief. These good results are in keeping with other published experience; and yet the acceptance of this syndrome as an entity has been strongly challenged. ${ }^{1314}$ The symptoms attributed to it are ill-defined and highly subjective; case reports abound with descriptions of associated disorders, previous surgical procedures, and continuing psychiatric problems. The only physical sign is of doubtful clinical value. A survey of 200 healthy adults ${ }^{15}$ showed epigastric bruits in $6.5 \%$, and only one of these had dyspepsia. More important, not only has incidental narrowing of the coeliac artery frequently been shown in symptom-free people by angiography, but it is also found in $44 \%$ of unselected cadavers examined. ${ }^{16}$ Finally, ligating the coeliac axis has been advocated as part of the surgical management of gastric cancer, and Appleby ${ }^{17}$ reported 13 patients so treated without any subsequent ischaemic problems.

This sort of evidence has to be disregarded by defenders of the legitimacy of the coeliac axis syndrome. Possibly ischaemic symptoms may result from diversion of blood flow into the coeliac axis from the superior mesenteric artery via the gastroduodenal network ${ }^{15}$-another steal syndrome-but small bowel function appears to be normal in these individuals, and restoration of flow pattern to normal does not relieve symptoms. ${ }^{15}$ There remains the problem of aetiology. At operation Watson and Sadikali ${ }^{12}$ noted that it was difficult to decide whether the median arcuate ligament alone, periarterial fibrous tissue, or the coeliac ganglion was responsible for the compression. In most of their cases not only did they divide the ligament but they also transected or excised the ganglion. Possibly, therefore, the success of operation depends more on autonomic neurectomy than decompression of the coeliac artery.

So where do we stand? The coincidence of some illdefined abdominal symptoms and narrowing of the coeliac axis does not add up to a recognisable disease entity. Any syndrome which provokes such vigorous argument must be regarded with scepticism; but, as Sleisenger ${ }^{14}$ has observed, it is difficult if not unfair to doubt that some of these patients are helped by surgery. Even so, the validity of this syndrome can be established only by further critical study. If we could show that patients with isolated coeliac axis compression who obtain permanent relief from surgical treatment have small bowel dysfunction, it would enormously strengthen acceptance of this apparent radiologically genuine problem $^{8}$ into routine clinical medicine.

${ }^{1}$ Marston, A, Clinics in Gastroenterology, 1972, 1, 539.

2 Fry, W J, and Kraft, R O, Surgery, Gynecology and Obstetrics, 1962, 117, 417.

${ }^{3}$ Rob, C G, Archives of Surgery, 1966, 93, 21.

${ }^{4}$ Michels, N A, Blood Supply and Anatomy of the Upper Abdominal Organs. Philadelphia, Lippincott, 1955.

${ }^{5}$ Harjola, P T, Annales Chirurgiae et Gynaecologiae Fenniae, 1963, 52, 547.

${ }^{6}$ Dunbar, J D, et al, American fournal of Roentgenology, 1965, 95, 731.

'Marable, S AL, Molnar, W, and Beman, F M, American fournal of Surgery, 1966, 111, 493.

${ }^{8}$ Herlinger, H, Clinics in Gastroenterology, 1972, 1, 547.

${ }^{9}$ Snyder, M A, Mahoney, E B, and Rob, C G, Surgery, 1967, 61, 372.

10 Stoney, R J, and Wylie, E J, Annals of Surgery, 1966, 164, 714

11 Lord, R S A, Stoney, R J, and Wylie, E J, Lancet, 1968, 2, 795.

12 Watson, W C, and Sadikali, F, Annals of Internal Medicine, 1977, 86, 278

${ }^{13}$ Drapanas, T, and Bron, K M, Annals of Surgery, 1966, 164, 1085.

${ }^{14}$ Sleisenger, M H, Annals of Internal Medicine, 1977, 86, 355.

${ }_{15}$ Edwards, A J, et al, British Medical fournal, 1970, 1, 342.

${ }^{16}$ Derrick, J R, Pollard, H S, and Moore, R M, Annals of Surgery, 1959, 149, 684.

17 Appleby, L H, Cancer, 1953, 6, 704.

\section{Why does coronary heart disease run in families?}

Chance alone will produce impressive clusters of a common disease, and chance rather than hereditary taint must underlie many a bad family history. In coronary heart disease (CHD), however, familial clustering is too strong to be ascribed wholly to chance. ${ }^{1-3}$ A positive family history should be recognised as a major risk factor-and one moreover which especially concerns the patient.

How can we distinguish a truly bad-risk family from a chance concatenation? Does clustering depend on the presence of identifiable and perhaps modifiable risk factors? Some is certainly due to shared genes, ${ }^{4}$ but how much can we blame on the more amenable influence of a shared environment? These questions will influence our advice to the unfortunate individual with a bad family history of heart disease, and some of the answers emerge from a recent Finnish report by Rissanen and Nikkila. ${ }^{5}$

These authors began by looking at the electrocardiographs of some 6000 workers in the Finnish wood and paper industry. In 750 men aged under 56 there were ischaemic changes, and 104 of these men were considered also to have angina. These 104 cases, with a matched control group, formed the probands for a family study. Half came from the east of Finland, where the incidence of CHD is the highest in the world, and the rest were from the south. The cases and controls had 1058 surviving first-degree relatives, who were assessed by a wellvalidated mailed questionnaire and by a simple physical examination at the local clinic. Death certificates were obtained for $93 \%$ of the 421 dead relatives.

The expected familial clustering of CHD was confirmed, but it was not uniform. The increase in risk was fourfold among brothers of the men with $\mathrm{CHD}$, but only twofold among fathers and apparently none at all among mothers. Relative risk is always an inadequate guide to counselling, for which we need to know the absolute risk faced, for instance, by a man whose brother has just had a heart attack. In Finland at least this is remarkably high: in such a man the probability of $\mathrm{CHD}$ 
appearing before the age of 65 was $65 \%$ in the eastern region and $52 \%$ in the south, compared with $31 \%$ and $5 \%$ respectively in brothers of healthy controls. These results suggest an apparent "ceiling effect," whereby the regional difference in CHD was almost obliterated among those with a positive family history. Conversely, the family history was much more ominous where the background risk was low: in this example the relative risk was about 6 in the south compared with only 1.3 in the high-risk eastern region.

How can we explain familial transmission? The main risk factors certainly run in families, partly through polygenic inheritance (factors such as blood pressure), rarely through the action of a single gene (familial hypercholesterolaemia), and in all cases additionally through a shared environment. Other investigators ${ }^{6}$ have concluded that aggregation of the known risk factors is only an incomplete explanation of the familial clustering of CHD, but the Finnish group disagree. They find very strong clustering of some major risk factors. For hyperlipoproteinaemia (mostly mixed) the relative risk was about 3 and for hypertension about 2, and the authors conclude that these factors accounted for most of the clustering of CHD. The implication is that (in Finland at least) a man with a family history of CHD has no increased risk unless he has a raised blood pressure or serum cholesterol concentration —or (particularly) both.

The extraordinarily high incidence of CHD in eastern Finland has always interested epidemiologists. The Seven Countries Study ${ }^{7}$ showed that it was to be expected from the high levels of risk factors which exist there, particularly the raised serum cholesterol concentrations. This new study extends this conclusion by showing that the big difference in incidence between the east and the south of Finland may again be accounted for by the east having twice the prevalence of hypertension and hyperlipoproteinaemia. We are glad to know that at least in Finland the familial and regional patterns of this disease seem to make sense.

1 Shanoff, H M, et al, Canadian Medical Association fournal, 1961, 84, 519. 2 Rose, G, British fournal of Preventive and Social Medicine, 1964, 18, 75.

3 Slack, J, and Evans, K A, Fournal of Medical Genetics, 1966, 3, 239.

4 Harvald, B, and Hauge, M, Acta Geneticae Medicae et Gemellologiae, $1970,19,248$.

${ }^{5}$ Rissanen, A M, and Nikkila, E A, British Heart fournal, 1977, 39, 875.

${ }^{6}$ Deutscher, S, Ostrander, L D, and Epstein, F H, American fournal of Epidemiology, 1970, 91, 233.

${ }^{7}$ Keys, A, Circulation, 1970, 41-42, suppl 1.

\section{Damaged laryngeal nerves in thyroid surgery}

Damage to the recurrent laryngeal nerve is occasionally an inevitable complication of thyroid surgery. It may be temporary and recover within three months or be permanent. Unilateral paralysis may cause few symptoms, with little alteration in the voice, but bilateral paralysis is a surgical catastrophe. Where the nerve was identified Lahey ${ }^{1}$ found an incidence of $0.3 \%$, but routine postoperative laryngoscopy was not carried out. With identification of the nerve and postoperative laryngoscopy the incidence has been variously reported as $0.6 \%,{ }^{2}$ Till $1.5 \%,{ }^{2}$ Wade $0 \%,{ }^{3}$ and Mountain et al $0 \cdot 27 .{ }^{4}$ When the nerve was not exposed and identified and postoperative laryngoscopy was carried out it rose to $4.5 \%$
(Blomstedt), ${ }^{5} 7 \%$ (Roy), ${ }^{6}$ and $9.5 \%$ in patients having a second operation on the gland (Beahrs). ${ }^{7}$

Most recently $\mathrm{Holt}^{8}$ has examined the problem again and has emphasised the importance of laryngoscopy both before and after operation. In 121 thyroid operations when the recurrent laryngeal nerve was identified either visually or by palpation the incidence of paralysis was $3 \%$, with a further $1 \%$ incidence of injury to the external laryngeal nerve. Preoperative laryngoscopy is essential in all patients but is particularly important in those who have had a previous operation on the gland and in carcinoma of the thyroid. Paralysis of one cord may be seen in patients with benign goitres. ${ }^{9}$ Immediate postoperative laryngoscopy (while the patient is in the operating theatre) is of value if the cords are seen to be moving normally, but pressure by the endotracheal tube or deep anaesthesia may prevent normal movement. Further postoperative laryngoscopy should be carried out within two weeks of the operation; this may identify many cases of unilateral paralysis which would not otherwise have been detected. ${ }^{6}$

Clearly, knowledge of the normal anatomy of the recurrent laryngeal nerve is essential. In a careful study Wade ${ }^{10}$ described eight anatomical variations in 100 thyroidectomies in the relation of the recurrent laryngeal nerve to the inferior thyroid artery: in $46 \%$ of cases the nerve was deep to the arterial trunk or its two divisions; in $10 \%$ it was superficial; while on the right side he identified no fewer than four anomalous nerves. Extralaryngeal branching is not uncommon: in more than one-third of cases the nerve divides into two or more branches. ${ }^{2}$

The nerve may be damaged at the lower pole when it is included in the ligature of the inferior thyroid veins or at the upper pole during haemostasis of the thyroid remnant. In the middle third of its course fixation by the branches of the inferior thyroid artery or enclosure by a nodule places the nerve at risk. Gross displacement of the nerve is common in large retrosternal goitres, when delivery of the gland into the neck will present a risk of damage to the nerve unless all the inferior thyroid veins have been divided first and the retrosternal prolongation of the gland carefully mobilised, when the nerve can then be identified.

The hazard of damage to the nerve can best be avoided by exposing and identifying it. The surgeon may palpate the nerve (it feels like a cord) and identify its position to help in the exposure; he should see it fully and display its relation to the inferior thyroid artery and its branches. This should be sufficient in most cases, but in those in which a previous operation has been carried out or where the surgeon is still in doubt about the nerve's identity a battery-operated stimulator $^{211}$ may be used to check the identity of the exposed nerve at operation. Laryngoscopy will show whether cords are moving or not, but if that is impossible owing to technical problems vibration of the cords may be detected by feeling the exposed thyroid cartilage. ${ }^{12}$.

Injury to the external laryngeal nerve is recognised less commonly. In $15 \%$ of cases Moosman found that the nerve was adherent to the superior thyroid artery and that in $6 \%$ it hooked around the suprapolar branches. ${ }^{13} \mathrm{He}$ suggested that the anterior branch of the superior thyroid artery should be ligated lower down on the upper pole and then the posterior branch tied separately. Holt ${ }^{8}$ also noted the importance of avoiding injury to this nerve, emphasising the disability that results from a combined recurrent laryngeal and external laryngeal nerve paralysis.

Riddell $^{2}$ found 55 cases of a permanent bilateral recurrent 